\title{
Superficial temporal muscle fascia grafting: Successful transplant of surgical induced necrotizing sclerosis
}

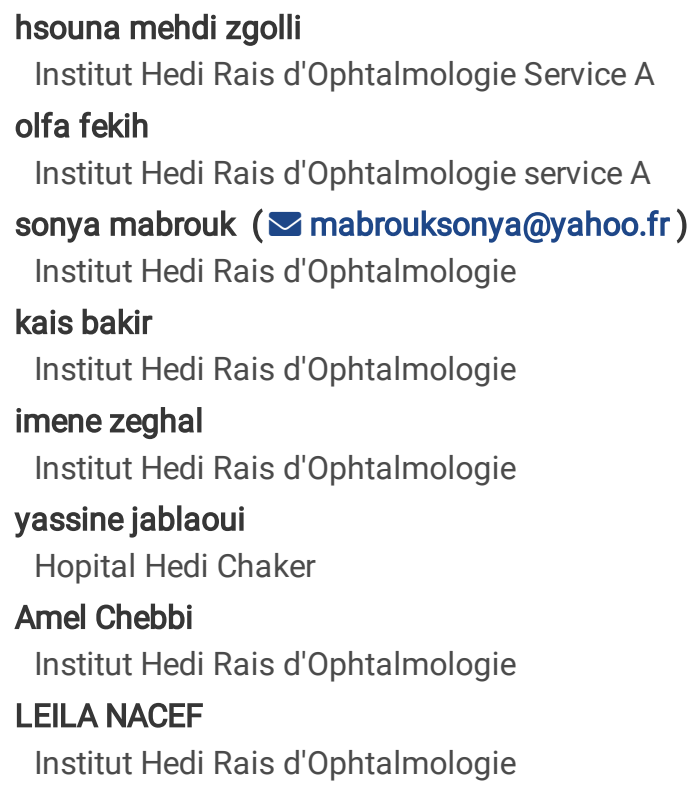




\section{Abstract}

Background To report the case of a surgical-induced necrotizing sclertitis (SINS) following vitreoretinal retinal surgery for rhegmatogenous retinal detachment, successfully managed by superficial muscle temporal fascia grafting. Case presentation An 18year-old teenage, with a history of a twenty-three gauges vitrectomy with silicone oil tamponade forrhegmatogenous retinal detachment of the left eye presented two months after surgery with intense left ocular pain, decreased visual acuity to counting fingers and eye redness. Split lamp exam showed: conjunctival infiltration with silicone oil, circumferential sclera thinningpredominantly in the superonasal quadrant with ectasia of the underling uvea). The fundus examination showed an attached retina. Necrotizing scleritis was the retained diagnosis. Performed etiological assessment was negative.Surgical induced necrotizing scleritis was the final diagnosis. An immunosuppressive therapy based on general corticoids was started. With the immanent risk of globe perforation, superficial muscle temporal fascia grafting was performed to cover the necrotizing sclera. The patient did well postoperatively without sclera thinning or ectasia and the fascia grafting still intact without retraction after six months of follow up. Conclusion This is the first case in the literature thatused the superficial temporal muscle fascia as a graft for sclera reinforcement in SINS. Wepropose a new support to reinforce the deficient sclera.This graft must be associated with prompt immunosuppressive therapy at high doses.

\section{Background}

Surgical Induced Necrotizing Sclerosis (SINS) is a rare and severe form of scleritis (1). It'sa postoperative complication reported after ocular surgery including cataract surgery, trabeculectomy, pterygium's excision, penetrating keratoplasty and pars plana vitrectomy $(2,3)$.

Exact pathogenesis of this disease is not yet fully understood nevertheless most likely theory is the immunological mechanism $(3,4)$.

The immunosuppressive therapy is the most successful and tolerated treatment $(2,3,5)$. However, this therapy especially in extended SINS, can be insufficient to control the progression's process. To the best of our knowledge the present case is the first one in literature reporting SINS management by superficial muscle temporal fascia grafting .

\section{Case Presentation}

An 18-year- old teenage, with myopiahistory, underwent a 23 gauges pars plana vitrectomy with silicone oil tamponade for rhematogenous retinal detachment of the left eye.Laser retinopexy of peripheral tears was intraoperatively conducted without cryotherapy. Two months later, the patient has suffered intense left ocular pain, decreased visual acuity and eye redness. Visual acuity was decreased to counting fingers. Split lamp exam showed: conjunctival infiltration with silicone oil, a circumferential scleral thinning, predominant in the superior nasal and ectasia of the underling uvea (figure 1a). Cornea was transparent, the photo motor reflex was normal with phacosclerosis. Fundus examination showed an attached retina, documented by the echography B.

In front of these signs, a necrotizingscleritis was the first evoked diagnosis. Postoperative infective scleritis was ruled out with negative microbiological assessment of scleral scrapping.Blood tests includingserologies (syphilis, tuberculosis, herpes, toxoplasmosis), full blood count, erythrocyte sedimentation rate, C-reactive protein, rheumatoid factor, antinuclear antibodyand anti-neutrophil cytoplasmic antibody tests were performed.

Ageneral physical examination was alsoperformed. Results were negative for both systemic vasculitis and arthritis. An orbit cerebral magnetic resonance imaging (MRI) was performed eliminating ocular or cerebral tumor.

Considering these findings, SINS was the final diagnosis.An immunosuppressive therapy based on general corticoids (prednisone 1 $\mathrm{mg} / \mathrm{kg}$ ) was started with topical corticosteroids. Owing to impending risk of globeperforation, superficial muscle temporal fascia grafting was performed wrapping thenecrotizing sclera (figure $1 \mathrm{~b}, \mathrm{c}, \mathrm{d}$ ). Oral azathioprine ( $2.5 \mathrm{mg} / \mathrm{kg}$ during the first month) was startedpostoperatively in addition to corticosteroid with progressive degression for both immusupressive therapies. The patient did well where the fascia grafting is still intact without retraction or rejection for6 months (figure 1e).

\section{Surgical technique:}

A 360-degree anterior conjunctival dissection was performed. We evacuated the silicone in subconjunctival. Exploration has revealed significant 360-degree scleral thinning, greater supra-nasalwithout obvious perforation (fig 1a, c). We measured the area of prolapse. Patches of aponevrosis of the superficial temporal muscle were cut and modeled according to the air of scleral prolapsed (fig $1 \mathrm{~b}, \mathrm{~d})$. The different patches had variable surfaces that werelarger than the areas of scleral defect ( $>1 \mathrm{~mm}$ minimum). The suture of the patchs was 
performed as follows: 4 separate cardinal points under tension, with a 10-0 monofilament, then a 360-degree overlock of each patch with a 6-0 vicryl yarn (fig 1d). We ended with a conjunctival closure on 360-degree by separated points by vicryl 7-0.

\section{Discussion}

SINS are a severe form of scleritis and a threat to globe integrity (1). It requires prompt and aggressive immunosuppressive therapy after ruling out infectious etiology $(1,2)$.

Many studies involved hypersensitivity response as the major mechanism of triggering SINS. Therefore, their therapeutic approachwas based on the here above involvement to treat SINS with immunosuppressive molecules $(2,3,4,9)$. Corticoids with high doses, cyclophosphamide and azathioprin are main immunosuppressive drugs used to control and stop SINS progression $(1,2,3,4)$.

In severe cases, in addition to immunosuppressive therapy, damaged tissue surgical replacement is required.The SINS'ssurgical managementis stillchallenging.Multiple material grafts have been used for patch grafting: amniotic membrane, pericardium, fascia lata, Gore-Tex (synthetic material) $(1,6)$.

The amniotic membrane is widely used for ocular surface reconstruction, especially in necrotizing scleritis with uveal ectasia. In fact,it's available, non-antigenicand without risk of immunologic rejection. Furthermore, the amniotic membrane has many growth factors, stimulates reepithalialization and reduces fibrosis and inflammation $(7,8)$.

Similarly, the fascia lata grafting shows promising results, especially in extended area of necrosis. $\mathrm{H}$. Kobtan reported a special case of SINS in a traumatic eye with multiple surgical interventions and multiple sclera damage. The fascia lata covered all the necrotizing area and provided the tectonic integrity of the globe (1). The fascia lata is relatively acellular, durable, with no risk of tissue reaction or rejectionand without risk of potentialdisease transmission (1).

To our knowledge, our case is unique in literature: It's the first case in the literature that used the superficial temporal muscle fascia as a graft for sclera reinforcement. We chose this tissue for several reasons: homograft technique, availability, acellular, sustainability, very strong and with large size. It successfully covered the hole ectasia, over 360-degree.

Inclusion of immunosuppressive therapy immediately on postoperative is crucial to avoid rejection and necrosis of the graft (10). In our case, the corticoids were administrated, with high doses before the surgery. Aziathioprin was immediatelyprescribedpostoperativelyin association to corticoids to increase graft survival.

\section{Conclusions}

SINS is a rare complication after pars plana vitrectomy with challenging management. Different approaches are proposed to control necrotizing extension and preserve globe integrity. Surgical management and aggressive immunotherapy are usually necessary in order to get successful results. In our case, we offera new support to reinforce the deficient sclera and reduce the uveal prolapsed: the superficial temporal muscle fascia providing a good tectonic support for the globe. This graft must be associated with prompt immunosuppressive therapy at high doses.

\section{Abbreviations}

SINS: surgical induced necroziting scleritis.

\section{Declarations}

Ethics approval and consent to participate

The study adhered to the tenets of the Declaration of Helsinki. It was approved by the local ethics committee and written informed consent was obtained from all participants before they recruited into the study.

Consent for publication

Written informed consent was obtained from the patient for publication of this study and any accompanying images.

Page $3 / 5$ 
Availability of Data and material:

Not applicable. Our manuscript present a case report, there is no data used to develop our case.

Competing interests

The authors declare that they have no competing interests.

Funding

No funding was obtained for this study.

Authors' contributions

There are no authors' contributions.

Acknowledgements

No applicable.

\section{References}

1. Kobtan $\mathrm{H}$. Use of autologous fascia lata as a natural biomaterial for tectonic support in surgically inducednecrotizing scleritis. Eye (Lond). 2015;29:580-4.

2. Morley AM, Pavesio C. Surgicallyinduced necrotising scleritis following three-port pars plana vitrectomy without scleralbuckling: a series of three cases. Eye (Lond).2008;22:162-4

3. Das S, Saurabh K, Biswas J. Postoperative necrotizing scleritis: a report of four cases.Middle East Afr J Ophthalmol. 2014;21(4):3503

4. Fong LP, Sainz de la Maza M, Rice BA, Kupferman AE, Foster CS. Immunopathology of scleritis. 1991;98:472-9.

5. Vagefi MR, Hollander DA, Seitzman GD, Margolis TP. Bilateral surgically induced necrotising scleritis with secondary superinfection. Br J Ophthalmol. 2005;89:124-5.

6. Zheng X, Kodama T, Goto T, Ohashi. Autologous fascia lata grafts for scleral repair in eyes with infectious necrotizing scleritis. Arch Ophthalmol.2011;129:1225-7.

7. Karalezli A, Kucukerdonmez C, Borazan M, Akova YA.

Successfultreatment of necrotizing scleritis after conjunctival autografting for pterygium with amniotic membrane Orbit. 2010;29:8890

8. Sangwan VS, Jain V, Gupta P. Structural and functional outcome of scleral patch graft.Eye (Lond). 2007;21:930-5

9. Diaz-Valle D, Benitez del Castillo JM, Castillo A,Sayagues O, Banares A, Garcia-Sanchez J. Immunologicand clinical evaluation of postsurgical necrotizing sclerocorneal ulceration. Cornea. 1998; 17: 371-375.

10. Wen JC, Lam J, Banitt MR. ScleralPatch Graft With

a Suture Reinforcement Technique in Surgical Management of Necrotizing Scleritis With Ectasia. 2018 Jul;37:933-935.

\section{Figures}





\section{Figure 1}

Legend: A: scleral thinningwith ectasia of the underling uvea. B: dissection of aponevosis of the superficial temporal muscle. C: ectasia of the uvea after conjonctival dissection: there is no sclera perforation. D: the temporal muscle was transplated over the sclera defect and sutured with interrupted 10-0 nylon sutures. E: 3months after the transplantation of the aponovrosis of the superficial temporal muscle.

\section{Supplementary Files}

This is a list of supplementary files associated with this preprint. Click to download.

- cheklist1.pdf 